\title{
Barnes-type Daehee with $\lambda$-parameter and degenerate Euler mixed-type polynomials
}

\author{
Dmitry V Dolgy', Dae San Kim², Taekyun Kim³ and Toufik Mansour ${ }^{4}$
}

\section{"Correspondence:}

dskim@sogang.ac.kr

${ }^{2}$ Department of Mathematics,

Sogang University, Seoul, 121-742, South Korea

Full list of author information is available at the end of the article

\begin{abstract}
In this paper, we consider the Barnes-type Daehee with $\lambda$-parameter and degenerate Euler mixed-type polynomials. We present several explicit formulas and recurrence relations for these polynomials. Also, we establish a connection between our polynomials and several known families of polynomials.
\end{abstract}

MSC: 05A15; 05A40; 11B83

Keywords: Barnes-type Daehee with $\lambda$-parameter and degenerate Euler mixed-type polynomial; umbral calculus

\section{Introduction}

In this paper, we use umbral calculus techniques (see $[1,2])$ to obtain several new and interesting identities of Barnes-type Daehee with $\lambda$-parameter and degenerate Euler mixedtype polynomials. To define the umbral calculus, let $\Pi$ be the algebra of polynomials in a single variable $x$ over $\mathbb{C}$ and $\Pi^{*}$ be the vector space of all linear functionals on $\Pi$. The action of a linear functional $L \in \Pi^{*}$ on a polynomial $p(x)$ is denoted by $\langle L \mid p(x)\rangle$, and linearly extended as $\left\langle c L+d L^{\prime} \mid p(x)\right\rangle=c\langle L \mid p(x)\rangle+d\left\langle L^{\prime} \mid p(x)\right\rangle$, where $c, d \in \mathbb{C}$. Define $\mathcal{H}=\left\{f(t)=\sum_{k \geq 0} a_{k} \frac{t^{k}}{k !} \mid a_{k} \in \mathbb{C}\right\}$ to be the algebra of formal power series in a single variable $t$. The formal power series $f(t) \in \mathcal{H}$ defines a linear functional on $\Pi$ by setting $\left\langle f(t) \mid x^{n}\right\rangle=a_{n}$ for all $n \geq 0$. Thus, we have (see $\left.[1,2]\right)$

$$
\left\langle t^{k} \mid x^{n}\right\rangle=n ! \delta_{n, k} \quad \text { for all } n, k \geq 0,
$$

where $\delta_{n, k}$ is the Kronecker symbol. Let $f_{L}(t)=\sum_{n \geq 0}\left\langle L \mid x^{n}\right\rangle \frac{t^{n}}{n !}$. By (1.1), we get that $\left\langle f_{L}(t) \mid x^{n}\right\rangle=\left\langle L \mid x^{n}\right\rangle$. Thus, the map $L \mapsto f_{L}(t)$ gives a vector space isomorphism from $\Pi^{*}$ onto $\mathcal{H}$. Therefore, $\mathcal{H}$ is thought of as a set of both formal power series and linear functionals, which is called the umbral algebra. The umbral calculus is the study of umbral algebra.

The order $O(f(t))$ of the non-zero power series $f(t)$ is defined to be $k$ when $f(t)=$ $\sum_{n \geq k} a_{n} t^{n}$ and $a_{k} \neq 0$. Suppose that $O(f(t))=1$ and $O(g(t))=0$. Then there exists a unique sequence $s_{n}(x)$ of polynomials such that $\left\langle g(t) f(t)^{k} \mid s_{n}(x)\right\rangle=n ! \delta_{n, k}$, where $n, k \geq 0$. The sequence $s_{n}(x)$ is called the Sheffer sequence for $(g(t), f(t))$, and we write $s_{n}(x) \sim(g(t), f(t))$ 
(see $[1,2])$. For $f(t) \in \mathcal{H}$ and $p(x) \in \Pi$, we have that $\left\langle e^{y t} \mid p(x)\right\rangle=p(y),\langle f(t) g(t) \mid p(x)\rangle=$ $\langle g(t) \mid f(t) p(x)\rangle, f(t)=\sum_{n \geq 0}\left\langle f(t) \mid x^{n}\right\rangle \frac{t^{n}}{n !}$ and $p(x)=\sum_{n \geq 0}\left\langle t^{n} \mid p(x)\right\rangle \frac{x^{n}}{n !}$. Therefore, $\left\langle t^{k} \mid p(x)\right\rangle=$ $p^{(k)}(0),\left\langle 1 \mid p^{(k)}(x)\right\rangle=p^{(k)}(0)$, where $p^{(k)}(0)$ denotes the $k$ th derivative of $p(x)$ with respect to $x$ at $x=0$. So, $t^{k} p(x)=p^{(k)}(x)=\frac{d^{k}}{d x^{k}} p(x)$ for all $k \geq 0$ (see $\left.[1,2]\right)$.

Let $s_{n}(x) \sim(g(t), f(t))$. Then we have

$$
\frac{1}{g(\bar{f}(t))} e^{y \bar{f}(t)}=\sum_{n \geq 0} s_{n}(y) \frac{t^{n}}{n !}
$$

for all $y \in \mathbb{C}$, where $\bar{f}(t)$ is the compositional inverse of $f(t)$ (see [1, 2]). For $s_{n}(x) \sim(g(t), f(t))$ and $r_{n}(x) \sim(h(t), \ell(t))$, let $s_{n}(x)=\sum_{k=0}^{n} c_{n, k} r_{k}(x)$. Then we have

$$
c_{n, k}=\frac{1}{k !}\left\langle\frac{h(\bar{f}(t))}{g(\bar{f}(t))}(\ell(\bar{f}(t)))^{k} \mid x^{n}\right\rangle
$$

(see $[1,2])$.

Throughout the paper, let $r, s \in \mathbb{Z}_{>0}$, and let $\mathbf{a}=\left(a_{1}, a_{2}, \ldots, a_{r}\right), \mathbf{b}=\left(b_{1}, b_{2}, \ldots, b_{s}\right)$ with $a_{j}, b_{i} \neq 0$ for all $i, j$. We define the Barnes-type Daehee with $\lambda$-parameter and degenerate Euler mixed-type polynomials $D \mathcal{E}_{n}(\lambda, x \mid \mathbf{a} ; \mathbf{b})$ (for other Barnes-types, see [3-5]) as

$$
P_{r, s}(t)(1+\lambda t)^{\frac{x}{\lambda}}=\sum_{n \geq 0} D \mathcal{E}_{n}(\lambda, x \mid \mathbf{a} ; \mathbf{b}) \frac{t^{n}}{n !},
$$

where we define

$$
P_{r, s}(t)=\prod_{i=1}^{r}\left(\frac{\log (1+\lambda t)}{\lambda\left((1+\lambda t)^{\frac{a_{i}}{\lambda}}-1\right)}\right) \prod_{i=1}^{s}\left(\frac{2}{(1+\lambda t)^{\frac{b_{i}}{\lambda}}+1}\right) .
$$

For $x=0, D \mathcal{E}_{n}(\lambda \mid \mathbf{a} ; \mathbf{b})=D \mathcal{E}_{n}(\lambda, 0 \mid \mathbf{a} ; \mathbf{b})$ are called the Barnes-type Daehee with $\lambda$-parameter and degenerate Euler mixed-type numbers.

We recall here that the polynomials $D_{n, \lambda}(x \mid \mathbf{a})$ given by

$$
P_{r, 0}(t)(1+\lambda t)^{\frac{x}{\lambda}}=\sum_{n \geq 0} D_{n, \lambda}(x \mid \mathbf{a}) \frac{t^{n}}{n !}
$$

are called the Barnes-type Daehee polynomials with $\lambda$-parameter (see [6, 7]). Also, the polynomials $\mathcal{E}_{n}(\lambda, x \mid \mathbf{b})$ given by

$$
P_{0, s}(t)(1+\lambda t)^{\frac{x}{\lambda}}=\sum_{n \geq 0} \mathcal{E}_{n}(\lambda, x \mid \mathbf{b}) \frac{t^{n}}{n !}
$$

are called the Barnes-type degenerate Euler polynomials which are studied in [8-11]. In the case $x=0$, we write $\mathcal{E}_{n}(\lambda \mid \mathbf{b})=\mathcal{E}_{n}(\lambda, 0 \mid \mathbf{b})$, which are called the Barnes-type degenerate Euler numbers. Note that $\lim _{\lambda \rightarrow 0} \mathcal{E}_{n}(\lambda, x \mid \mathbf{b})=E_{n}(x \mid \mathbf{b})$ and $\lim _{\lambda \rightarrow \infty} \lambda^{-n} \mathcal{E}_{n}(\lambda, \lambda x \mid \mathbf{b})=(x)_{n}$, where $(x)_{n}=\prod_{i=0}^{n-1}(x-i)$ with $(x)_{0}=1$ and $E_{n}(x \mid \mathbf{b})$ are the Barnes-type degenerate Euler polynomials given by

$$
\prod_{i=1}^{s}\left(\frac{2}{e^{b_{i} t}+1}\right) e^{x t}=\sum_{n \geq 0} E_{n}(x \mid \mathbf{b}) \frac{t^{n}}{n !} .
$$


It is immediate from (1.2) and (1.4) to see that $D \mathcal{E}_{n}(\lambda, x \mid \mathbf{a} ; \mathbf{b})$ is the Sheffer sequence for the pair $g(t)=\prod_{i=1}^{r}\left(\frac{e^{a_{i} t}-1}{t}\right) \prod_{i=1}^{s}\left(\frac{e^{b_{i} t}+1}{2}\right)$ and $f(t)=\frac{e^{\lambda t}-1}{\lambda}$. Thus,

$$
D \mathcal{E}_{n}(\lambda, x \mid \mathbf{a} ; \mathbf{b}) \sim\left(\prod_{i=1}^{r}\left(\frac{e^{a_{i} t}-1}{t}\right) \prod_{i=1}^{s}\left(\frac{e^{b_{i} t}+1}{2}\right), \frac{e^{\lambda t}-1}{\lambda}\right) .
$$

The aim of the present paper is to present several new identities for Barnes-type Daehee with $\lambda$-parameter and degenerate Euler mixed-type polynomials by the use of umbral calculus. For some of the related works, one is referred to the papers [12-20].

\section{Explicit formulas}

In this section we suggest several explicit formulas for the Barnes-type Daehee with $\lambda$-parameter and degenerate Euler mixed-type polynomials. To do that, we recall that the Stirling numbers $S_{1}(n, m)$ of the first kind are defined as $(x)_{n}=\sum_{m=0}^{n} S_{1}(n, m) x^{m} \sim\left(1, e^{t}-1\right)$ or $\frac{1}{j !}(\log (1+t))^{j}=\sum_{\ell \geq j} S_{1}(\ell, j) \frac{t^{\ell}}{\ell !}$. Let $(x \mid \lambda)_{n}$ be the generalized falling factorials defined by $(x \mid \lambda)_{n}=\prod_{i=0}^{n-1}(x-i \lambda)$ with $(x \mid \lambda)_{0}=1$, namely $(x \mid \lambda)_{n}=\lambda^{n}(x / \lambda)_{n}$.

Let $B E_{n}(x \mid \mathbf{a} ; \mathbf{b})$ be the Barnes-type Bernoulli and Euler mixed-type polynomials given by

$$
\prod_{i=1}^{r}\left(\frac{t}{e^{a_{i} t}-1}\right) \prod_{i=1}^{s}\left(\frac{2}{e^{b_{i} t}+1}\right) e^{x t}=\sum_{n \geq 0} B E_{n}(x \mid \mathbf{a} ; \mathbf{b}) \frac{t^{n}}{n !} .
$$
Note that $B E_{n}^{r, s}(x)$ denotes the special case $B E_{n}(x \mid \underbrace{1,1, \ldots, 1}_{r} ; \underbrace{1,1, \ldots, 1}_{s})$ and was treated in
$[21,22]$ by using $p$-adic integrals on $\mathbb{Z}_{p}$.

Theorem 2.1 For all $n \geq 0$,

$$
D \mathcal{E}_{n}(\lambda, x \mid \mathbf{a} ; \mathbf{b})=\sum_{m=0}^{n} S_{1}(n, m) \lambda^{n-m} B E_{m}(x \mid \mathbf{a} ; \mathbf{b}) .
$$

Proof By (1.6), we have that

$$
\prod_{i=1}^{r}\left(\frac{e^{a_{i} t}-1}{t}\right) \prod_{i=1}^{s}\left(\frac{e^{b_{i} t}+1}{2}\right) D \mathcal{E}_{n}(\lambda, x \mid \mathbf{a} ; \mathbf{b}) \sim\left(1, \frac{e^{\lambda t}-1}{\lambda}\right) .
$$

Thus,

$$
\begin{aligned}
D \mathcal{E}_{n}(\lambda, x \mid \mathbf{a} ; \mathbf{b}) & =\sum_{m=0}^{n} S_{1}(n, m) \lambda^{n-m} \prod_{i=1}^{r}\left(\frac{t}{e^{a_{i} t}-1}\right) \prod_{i=1}^{s}\left(\frac{2}{e^{b_{i} t}+1}\right) x^{m} \\
& =\sum_{m=0}^{n} S_{1}(n, m) \lambda^{n-m} B E_{m}(x \mid \mathbf{a} ; \mathbf{b}),
\end{aligned}
$$

as claimed.

Theorem 2.2 For all $n \geq 0$,

$$
D \mathcal{E}_{n}(\lambda, x \mid \mathbf{a} ; \mathbf{b})=\sum_{j=0}^{n}\left(\sum_{\ell=j}^{n}\left(\begin{array}{l}
n \\
\ell
\end{array}\right) S_{1}(\ell, j) \lambda^{\ell-j} D \mathcal{E}_{n-\ell}(\lambda \mid \mathbf{a} ; \mathbf{b})\right) x^{j} .
$$


Proof We proceed the proof by applying the conjugate representation: for $s_{n}(x) \sim$ $(g(t), f(t))$, we have $S_{n}(x)=\sum_{j=0}^{n} \frac{1}{j !}\left\langle g(\bar{f}(t))^{-1} \bar{f}(t)^{j} \mid x^{n}\right\rangle x^{j}$. By (1.6), we obtain

$$
\begin{aligned}
& \left\langle g(\bar{f}(t))^{-1} \bar{f}(t)^{j} \mid x^{n}\right\rangle \\
& \quad=\left\langle P_{r, s}(t) \frac{\log ^{j}(1+\lambda t)}{\lambda^{j}} \mid x^{n}\right\rangle=\lambda^{-j}\left\langle P_{r, s}(t) \mid j ! \sum_{\ell \geq j} S_{1}(\ell, j) \frac{\lambda^{\ell} t^{\ell}}{\ell !} x^{n}\right\rangle \\
& \quad=\lambda^{-j} j ! \sum_{\ell=j}^{n}\left(\begin{array}{l}
n \\
\ell
\end{array}\right) S_{1}(\ell, j) \lambda^{\ell}\left\langle P_{r, s}(t) \mid x^{n-\ell}\right\rangle=\lambda^{-j} j ! \sum_{\ell=j}^{n}\left(\begin{array}{l}
n \\
\ell
\end{array}\right) S_{1}(\ell, j) \lambda^{\ell} D \mathcal{E}_{n-\ell}(\lambda \mid \mathbf{a} ; \mathbf{b}) .
\end{aligned}
$$

Therefore, $D \mathcal{E}_{n}(\lambda, x \mid \mathbf{a} ; \mathbf{b})=\sum_{j=0}^{n}\left(\sum_{\ell=j}^{n}\left(\begin{array}{l}n \\ \ell\end{array}\right) S_{1}(\ell, j) \lambda^{\ell-j} D \mathcal{E}_{n-\ell}(\lambda \mid \mathbf{a} ; \mathbf{b})\right) x^{j}$, as claimed.

Theorem 2.3 For all $n \geq 1$,

$$
D \mathcal{E}_{n}(\lambda, x \mid \mathbf{a} ; \mathbf{b})=\sum_{\ell=0}^{n-1}\left(\begin{array}{c}
n-1 \\
\ell
\end{array}\right) \lambda^{\ell} B_{\ell}^{(n)} B E_{n-\ell}(x \mid \mathbf{a} ; \mathbf{b})
$$

where $B_{\ell}^{(n)}$ is the $\ell$ th Bernoulli number of order $n$ (see [23]).

Proof We proceed the proof by using the following transfer formula: for $p_{n}(x) \sim(1, f(t))$ and $q_{n}(x) \sim(1, g(t))$, we have that $q_{n}(x)=x\left(\frac{f(t)}{g(t)}\right)^{n} x^{-1} p_{n}(x)$ for all $n \geq 1$. So, by the fact that $x^{n} \sim(1, t)$ and $(2.2)$, we obtain

$$
\begin{aligned}
& \prod_{i=1}^{r}\left(\frac{e^{a_{i} t}-1}{t}\right) \prod_{i=1}^{s}\left(\frac{e^{b_{i} t}+1}{2}\right) D \mathcal{E}_{n}(\lambda, x \mid \mathbf{a} ; \mathbf{b}) \\
& \quad=x\left(\frac{\lambda t}{e^{\lambda t}-1}\right)^{n} x^{n-1}=x \sum_{\ell \geq 0} B_{\ell}^{(n)} \frac{\lambda^{\ell} t^{\ell}}{\ell !} x^{n-1}=\sum_{\ell=0}^{n-1}\left(\begin{array}{c}
n-1 \\
\ell
\end{array}\right) \lambda^{\ell} B_{\ell}^{(n)} x^{n-\ell},
\end{aligned}
$$

which, by (2.1), implies

$$
\begin{aligned}
D \mathcal{E}_{n}(\lambda, x \mid \mathbf{a} ; \mathbf{b}) & =\sum_{\ell=0}^{n-1}\left(\begin{array}{c}
n-1 \\
\ell
\end{array}\right) \lambda^{\ell} B_{\ell}^{(n)} \prod_{i=1}^{r}\left(\frac{t}{e^{a_{i} t}-1}\right) \prod_{i=1}^{s}\left(\frac{2}{e^{b_{i} t}+1}\right) x^{n-\ell} \\
& =\sum_{\ell=0}^{n-1}\left(\begin{array}{c}
n-1 \\
\ell
\end{array}\right) \lambda^{\ell} B_{\ell}^{(n)} B E_{n-\ell}(x \mid \mathbf{a} ; \mathbf{b}),
\end{aligned}
$$

as required.

In order to state our next theorem, we recall the polynomials $\beta \mathcal{E}_{n}(\lambda, x \mid \mathbf{a} ; \mathbf{b})$, which are called the Barnes-type degenerate Bernoulli and Euler mixed-type polynomials. They are defined as

$$
Q_{r, s}(t)(1+\lambda t)^{\frac{x}{\lambda}}=\sum_{n \geq 0} \beta \mathcal{E}_{n}(\lambda, x \mid \mathbf{a} ; \mathbf{b}) \frac{t^{n}}{n !}
$$

where $Q_{r, s}(t)=\prod_{i=1}^{r}\left(\frac{t}{(1+\lambda t)^{\frac{a_{i}}{\lambda}}-1}\right) \prod_{i=1}^{s}\left(\frac{2}{(1+\lambda t)^{\frac{b_{i}}{\lambda}+1}}\right)$, for example, see [3]. 
Theorem 2.4 For all $n \geq 0$,

$$
D \mathcal{E}_{n}(\lambda, x \mid \mathbf{a} ; \mathbf{b})=\sum_{\ell=0}^{n} \frac{\left(\begin{array}{c}
n \\
\ell
\end{array}\right)}{\left(\begin{array}{c}
+r \\
r
\end{array}\right)} \lambda^{\ell} S_{1}(\ell+r, r) \beta \mathcal{E}_{n-\ell}(\lambda, x \mid \mathbf{a} ; \mathbf{b}) .
$$

Proof By (1.4), we have

$$
\begin{aligned}
D \mathcal{E}_{n}(\lambda, y \mid \mathbf{a} ; \mathbf{b}) & =\left\langle\sum_{\ell \geq 0} D \mathcal{E}_{\ell}(\lambda, y \mid \mathbf{a} ; \mathbf{b}) \frac{t^{\ell}}{\ell !} \mid x^{n}\right\rangle=\left\langle P_{r, s}(t)(1+\lambda t)^{\frac{y}{\lambda}} \mid x^{n}\right\rangle \\
& =\left\langle Q_{r, s}(t)(1+\lambda t)^{\frac{y}{\lambda}} \mid \frac{\log ^{r}(1+\lambda t)}{\lambda^{r} t^{r}} x^{n}\right\rangle \\
& =\left\langle Q_{r, s}(t)(1+\lambda t)^{\frac{y}{\lambda}} \mid r ! \sum_{\ell \geq 0} \frac{S_{1}(\ell+r, r) \lambda^{\ell} t^{\ell}}{(\ell+r) !} x^{n}\right\rangle \\
& =\sum_{\ell=0}^{n} \frac{\left(\begin{array}{c}
n \\
\ell
\end{array}\right)}{\left(\begin{array}{c}
\ell+r \\
r
\end{array}\right)} \lambda^{\ell} S_{1}(\ell+r, r)\left\langle\sum_{m \geq 0} \beta \mathcal{E}_{m}(\lambda, y \mid \mathbf{a} ; \mathbf{b}) \frac{t^{m}}{m !} \mid x^{n-\ell}\right\rangle,
\end{aligned}
$$

which, by (2.3), implies $D \mathcal{E}_{n}(\lambda, x \mid \mathbf{a} ; \mathbf{b})=\sum_{\ell=0}^{n} \frac{\left(\begin{array}{c}n \\ \ell\end{array}\right)}{\left(\begin{array}{c}\ell+r \\ r\end{array}\right)} \lambda^{\ell} S_{1}(\ell+r, r) \beta \mathcal{E}_{n-\ell}(\lambda, x \mid \mathbf{a} ; \mathbf{b})$, as required.

In order to present our next theorem, we recall the polynomials $\beta_{n}(\lambda, x \mid \mathbf{a})$, which are called the Barnes-type degenerate Bernoulli polynomials. They are given by

$$
Q_{r, 0}(t)(1+\lambda t)^{\frac{x}{\lambda}}=\sum_{n \geq 0} \beta_{n}(\lambda, x \mid \mathbf{a}) \frac{t^{n}}{n !},
$$

for example, see $[8,9,23]$.

Theorem 2.5 For all $n \geq 0$,

$$
\begin{aligned}
D \mathcal{E}_{n}(\lambda, x \mid \mathbf{a} ; \mathbf{b}) & =\sum_{\ell=0}^{n} \sum_{m=0}^{n-\ell} \frac{\left(\begin{array}{c}
n \\
\ell
\end{array}\right)\left(\begin{array}{c}
n-\ell \\
m
\end{array}\right)}{\left(\begin{array}{c}
\ell+r \\
r
\end{array}\right)} \lambda^{\ell} S_{1}(\ell+r, r) \mathcal{E}_{n-\ell-m}(\lambda \mid \mathbf{b}) \beta_{m}(\lambda, x \mid \mathbf{a}) \\
& =\sum_{\ell=0}^{n} \sum_{m=0}^{n-\ell} \frac{\left(\begin{array}{c}
n \\
\ell
\end{array}\right)\left(\begin{array}{c}
n-\ell \\
m
\end{array}\right)}{\left(\begin{array}{c}
\ell+r \\
r
\end{array}\right)} \lambda^{\ell} S_{1}(\ell+r, r) \beta_{n-\ell-m}(\lambda \mid \mathbf{a}) \mathcal{E}_{m}(\lambda, x \mid \mathbf{b}) .
\end{aligned}
$$

Proof By the proof of Theorem 2.4, we have

$$
\begin{aligned}
D \mathcal{E}_{n}(\lambda, y \mid \mathbf{a} ; \mathbf{b}) & =\sum_{\ell=0}^{n} \frac{\left(\begin{array}{c}
n \\
\ell
\end{array}\right)}{\left(\begin{array}{c}
\ell+r \\
r
\end{array}\right)} \lambda^{\ell} S_{1}(\ell+r, r)\left\langle Q_{r, s}(t)(1+\lambda t)^{\frac{y}{\lambda}} \mid x^{n-\ell}\right\rangle \\
& =\sum_{\ell=0}^{n} \frac{\left(\begin{array}{c}
n \\
\ell
\end{array}\right)}{\left(\begin{array}{c}
\ell+r \\
r
\end{array}\right)} \lambda^{\ell} S_{1}(\ell+r, r)\left\langle Q_{0, s}(t) \mid Q_{r, 0}(t)(1+\lambda t)^{\frac{y}{\lambda}} x^{n-\ell}\right\rangle .
\end{aligned}
$$

Thus, by (1.5) and (2.4), we obtain

$$
\begin{aligned}
& D \mathcal{E}_{n}(\lambda, y \mid \mathbf{a} ; \mathbf{b}) \\
& \quad=\sum_{\ell=0}^{n} \frac{\left(\begin{array}{c}
n \\
\ell
\end{array}\right)}{\left(\begin{array}{c}
\ell+r \\
r
\end{array}\right)} \lambda^{\ell} S_{1}(\ell+r, r)\left\langle Q_{0, s}(t) \mid \sum_{m=0}^{n-\ell}\left(\begin{array}{c}
n-\ell \\
m
\end{array}\right) \beta_{m}(\lambda, y \mid \mathbf{a}) x^{n-\ell-m}\right\rangle
\end{aligned}
$$




$$
\begin{aligned}
& =\sum_{\ell=0}^{n} \frac{\left(\begin{array}{c}
n \\
\ell
\end{array}\right)}{\left(\begin{array}{c}
\ell+r \\
r
\end{array}\right)} \lambda^{\ell} S_{1}(\ell+r, r) \sum_{m=0}^{n-\ell}\left(\begin{array}{c}
n-\ell \\
m
\end{array}\right) \beta_{m}(\lambda, y \mid \mathbf{a})\left\langle Q_{0, s}(t) \mid x^{n-\ell-m}\right\rangle \\
& =\sum_{\ell=0}^{n} \frac{\left(\begin{array}{c}
n \\
\ell
\end{array}\right)}{\left(\begin{array}{c}
\ell+r \\
r
\end{array}\right)} \lambda^{\ell} S_{1}(\ell+r, r) \sum_{m=0}^{n-\ell}\left(\begin{array}{c}
n-\ell \\
m
\end{array}\right) \beta_{m}(\lambda, y \mid \mathbf{a}) \mathcal{E}_{n-\ell-m}(\lambda \mid \mathbf{b}),
\end{aligned}
$$

which completes the proof of the first formula.

The second formula can be obtained by using very similar techniques.

\section{Recurrence relations}

In this section, we present several recurrence relations for Barnes-type Daehee with $\lambda$-parameter and degenerate Euler mixed-type polynomials. Our first recurrence is based on the polynomials $(x \mid \lambda)_{n}$.

Theorem 3.1 For all $n \geq 0$,

$$
D \mathcal{E}_{n}(\lambda, x+y \mid \mathbf{a} ; \mathbf{b})=\sum_{j=0}^{n}\left(\begin{array}{l}
n \\
j
\end{array}\right) D \mathcal{E}_{j}(\lambda, x \mid \mathbf{a} ; \mathbf{b})(y \mid \lambda)_{n-j} .
$$

Proof Let $p_{n}(x)=\prod_{i=1}^{r}\left(\frac{e^{a_{i} t}-1}{t}\right) \prod_{i=1}^{s}\left(\frac{e^{b_{i} t}+1}{2}\right) D \mathcal{E}_{n}(\lambda, x \mid \mathbf{a} ; \mathbf{b})$. By (2.2) we have that $p_{n}(x)=$ $(x \mid \lambda)_{n} \sim\left(1, \frac{e^{\lambda t}-1}{\lambda}\right)$, which leads to the required recurrence.

The second recurrence is obtained from the fact that $f(t) s_{n}(x)=n s_{n-1}(x)$ for all $s_{n}(x) \sim$ $(g(t), f(t))($ see $[1,2])$.

Theorem 3.2 For all $n \geq 1$,

$$
D \mathcal{E}_{n}(\lambda, x+\lambda \mid \mathbf{a} ; \mathbf{b})-D \mathcal{E}_{n}(\lambda, x \mid \mathbf{a} ; \mathbf{b})=n \lambda D \mathcal{E}_{n-1}(\lambda, x \mid \mathbf{a} ; \mathbf{b})
$$

Proof By (1.6) and $f(t) s_{n}(x)=n s_{n-1}(x)$ whenever $s_{n}(x) \sim(g(t), f(t))$, we have

$$
\frac{e^{\lambda t}-1}{\lambda} D \mathcal{E}_{n}(\lambda, x \mid \mathbf{a} ; \mathbf{b})=n D \mathcal{E}_{n-1}(\lambda, x \mid \mathbf{a} ; \mathbf{b}),
$$

which implies $D \mathcal{E}_{n}(\lambda, x+\lambda \mid \mathbf{a} ; \mathbf{b})-D \mathcal{E}_{n}(\lambda, x \mid \mathbf{a} ; \mathbf{b})=n \lambda D \mathcal{E}_{n-1}(\lambda, x \mid \mathbf{a} ; \mathbf{b})$, as required.

The next result gives an explicit formula for $\frac{d}{d x} D \mathcal{E}_{n}(\lambda, x+\lambda \mid \mathbf{a} ; \mathbf{b})$.

Theorem 3.3 For all $n \geq 1$,

$$
\frac{d}{d x} D \mathcal{E}_{n}(\lambda, x \mid \mathbf{a} ; \mathbf{b})=n ! \sum_{\ell=0}^{n-1} \frac{(-\lambda)^{n-\ell-1}}{\ell !(n-\ell)} D \mathcal{E}_{\ell}(\lambda, x \mid \mathbf{a} ; \mathbf{b})
$$

Proof It is well known that for $s_{n}(x) \sim(g(t), f(t)), \frac{d}{d x} s_{n}(x)=\sum_{\ell=0}^{n-1}\left(\begin{array}{l}n \\ \ell\end{array}\right)\left\langle\bar{f}(t) \mid x^{n-\ell}\right\rangle s_{\ell}(x)$ (see [1, $2])$. In our case, by (1.6), we have

$$
\begin{aligned}
\left\langle\bar{f}(t) \mid x^{n-\ell}\right\rangle & =\left\langle\frac{1}{\lambda} \log (1+\lambda t) \mid x^{n-\ell}\right\rangle \\
& =\lambda^{-1}\left\langle\sum_{m \geq 1} \frac{(-1)^{m-1}(m-1) ! \lambda^{m} t^{m}}{m !} \mid x^{n-\ell}\right\rangle
\end{aligned}
$$




$$
\begin{aligned}
& =\lambda^{-1}(-1)^{n-\ell-1} \lambda^{n-\ell}(n-\ell-1) ! \\
& =(-\lambda)^{n-\ell-1}(n-\ell-1) !
\end{aligned}
$$

Thus $\frac{d}{d x} D \mathcal{E}_{n}(\lambda, x \mid \mathbf{a} ; \mathbf{b})=n ! \sum_{\ell=0}^{n-1} \frac{(-\lambda)^{n-\ell-1}}{\ell !(n-\ell)} D \mathcal{E}_{\ell}(\lambda, x \mid \mathbf{a} ; \mathbf{b})$, as required.

Another recurrence relation can be stated as follows.

Theorem 3.4 For all $n \geq 1$,

$$
\begin{aligned}
& D \mathcal{E}_{n}(\lambda, x \mid \mathbf{a} ; \mathbf{b}) \\
& =\left(x-\sum_{i=1}^{r} a_{i}-\sum_{j=1}^{s} b_{j}\right) D \mathcal{E}_{n-1}(\lambda, x-\lambda \mid \mathbf{a} ; \mathbf{b})+\frac{r}{n} \sum_{\ell=0}^{n}\left(\begin{array}{l}
n \\
\ell
\end{array}\right) \lambda^{\ell} \mathfrak{b}_{\ell} D \mathcal{E}_{n-\ell}(\lambda, x-\lambda \mid \mathbf{a} ; \mathbf{b}) \\
& \quad-\frac{1}{n} \sum_{i=1}^{r} a_{i} \sum_{\ell=0}^{n}\left(\begin{array}{l}
n \\
\ell
\end{array}\right) \lambda^{\ell} \mathfrak{b}_{\ell} D \mathcal{E}_{n-\ell}\left(\lambda, x-\lambda \mid a_{i}, a_{1}, \ldots, a_{r} ; \mathbf{b}\right) \\
& \quad+\frac{1}{2} \sum_{j=1}^{s} b_{j} D \mathcal{E}_{n-1}\left(\lambda, x-\lambda \mid \mathbf{a} ; b_{j}, b_{1}, \ldots, b_{s}\right),
\end{aligned}
$$

where $\mathfrak{b}_{n}$ is the nth Bernoulli number of the second kind, which is defined by $\frac{t}{\log (1+t)}=$ $\sum_{n \geq 0} \mathfrak{b}_{n} \frac{t^{n}}{n !}$.

Proof Let $n \geq 1$. Then

$$
\begin{aligned}
D \mathcal{E}_{n}(\lambda, y \mid \mathbf{a} ; \mathbf{b}) & \\
= & \left\langle\sum_{\ell \geq 0} D \mathcal{E}_{\ell}(\lambda, y \mid \mathbf{a} ; \mathbf{b}) \frac{t^{\ell}}{\ell !} \mid x^{n}\right\rangle \\
= & \left\langle P_{r, s}(t)(1+\lambda t)^{y / \lambda} \mid x^{n}\right\rangle=\left\langle\frac{d}{d t}\left(P_{r, s}(t)(1+\lambda t)^{y / \lambda}\right) \mid x^{n-1}\right\rangle \\
= & \frac{d}{d t} \prod_{i=1}^{r}\left(\frac{\log (1+\lambda t)}{\lambda\left((1+\lambda t)^{\frac{a_{i}}{\lambda}}-1\right)}\right) \prod_{i=1}^{s}\left(\frac{2}{(1+\lambda t)^{\frac{b_{i}}{\lambda}}+1}\right)(1+\lambda t)^{y / \lambda}\left|x^{n-1}\right\rangle \\
& +\left\langle\prod_{i=1}^{r}\left(\frac{\log (1+\lambda t)}{\lambda\left((1+\lambda t)^{\frac{a_{i}}{\lambda}}-1\right)}\right) \frac{d}{d t} \prod_{i=1}^{s}\left(\frac{2}{(1+\lambda t)^{\frac{b_{i}}{\lambda}}+1}\right)(1+\lambda t)^{y / \lambda} \mid x^{n-1}\right\rangle \\
& +\left\langle P_{r, s}(t) \frac{d}{d t}(1+\lambda t)^{y / \lambda} \mid x^{n-1}\right\rangle .
\end{aligned}
$$

By (1.6), the term in (3.3) equals

$$
y\left\langle P_{r, s}(t)(1+\lambda t)^{(y-\lambda) / \lambda} \mid x^{n-1}\right\rangle=y D \mathcal{E}_{n-1}(\lambda, y-\lambda \mid \mathbf{a} ; \mathbf{b}) .
$$

For the term in (3.2), we observe that

$$
\frac{d}{d t} \prod_{i=1}^{s}\left(\frac{2}{(1+\lambda t)^{\frac{b_{i}}{\lambda}}+1}\right)=\prod_{i=1}^{s}\left(\frac{2}{(1+\lambda t)^{\frac{b_{i}}{\lambda}}+1}\right) \sum_{i=1}^{s}\left(\frac{-b_{i}}{1+\lambda t}+\frac{b_{i}}{2(1+\lambda t)} \frac{2}{(1+\lambda t)^{b_{i} / \lambda}+1}\right) .
$$


So the term in (3.2) is

$$
\begin{gathered}
-\sum_{j=1}^{s} b_{j}\left\langle P_{r, s}(t)(1+\lambda t)^{(y-\lambda) / \lambda} \mid x^{n-1}\right\rangle+\frac{1}{2} \sum_{j=1}^{s} b_{j}\left\langle P_{r, s}(t) \frac{2(1+\lambda t)^{(y-\lambda) / \lambda}}{(1+\lambda t)^{b_{j} / \lambda}+1} \mid x^{n-1}\right\rangle \\
=-\sum_{j=1}^{s} b_{j} D \mathcal{E}_{n-1}(\lambda, y-\lambda \mid \mathbf{a} ; \mathbf{b})+\frac{1}{2} \sum_{j=1}^{s} b_{j} D \mathcal{E}_{n-1}\left(\lambda, y-\lambda \mid \mathbf{a} ; b_{j}, b_{1}, \ldots, b_{s}\right)
\end{gathered}
$$

For the term in (3.1), we note that

$$
\begin{aligned}
(1 & +\lambda t) \frac{d}{d t} \prod_{i=1}^{r}\left(\frac{\log (1+\lambda t)}{\lambda\left((1+\lambda t)^{\frac{a_{i}}{\lambda}}-1\right)}\right) \\
& =\prod_{i=1}^{r}\left(\frac{\log (1+\lambda t)}{\lambda\left((1+\lambda t)^{\frac{a_{i}}{\lambda}}-1\right)}\right)\left(-\sum_{i=1}^{r} a_{i}+\frac{1}{t} \sum_{i=1}^{r}\left(\frac{\lambda t}{\log (1+\lambda t)}-\frac{a_{i} t}{(1+\lambda t)^{a_{i} / \lambda}-1}\right)\right),
\end{aligned}
$$

where $\frac{\lambda t}{\log (1+\lambda t)}-\frac{a_{i} t}{(1+\lambda t)^{a_{i} / \lambda}-1}$ has order at least 1 . Thus, the term in (3.1) equals

$$
\begin{aligned}
-\sum_{i=1}^{r} & a_{i}\left\langle P_{r, s}(t)(1+\lambda t)^{(y-\lambda) / \lambda} \mid x^{n-1}\right\rangle \\
& +\left\langle P_{r, s}(t)(1+\lambda t)^{(y-\lambda) / \lambda} \mid \frac{1}{t} \sum_{i=1}^{r}\left(\frac{\lambda t}{\log (1+\lambda t)}-\frac{a_{i} t}{(1+\lambda t)^{a_{i} / \lambda}-1}\right) x^{n-1}\right\rangle \\
= & -\sum_{i=1}^{r} a_{i} D \mathcal{E}_{n-1}(\lambda, y-\lambda \mid \mathbf{a} ; \mathbf{b}) \\
& +\frac{1}{n}\left\langle P_{r, s}(t)(1+\lambda t)^{(y-\lambda) / \lambda} \mid \sum_{i=1}^{r}\left(\frac{\lambda t}{\log (1+\lambda t)}-\frac{a_{i} t}{(1+\lambda t)^{a_{i} / \lambda}-1}\right) x^{n}\right\rangle \\
= & -\sum_{i=1}^{r} a_{i} D \mathcal{E}_{n-1}(\lambda, y-\lambda \mid \mathbf{a} ; \mathbf{b}) \\
& +\frac{r}{n}\left\langle P_{r, s}(t)(1+\lambda t)^{(y-\lambda) / \lambda} \mid \sum_{\ell \geq 0}^{r} \mathfrak{b}_{\ell} \frac{\lambda^{\ell} t^{\ell}}{\ell !} x^{n}\right\rangle \\
& -\frac{1}{n} \sum_{i=1}^{r} a_{i}\left\langle\frac{\log (1+\lambda t)}{\lambda\left((1+\lambda t)^{a_{i} / \lambda}-1\right)} P_{r, s}(t)(1+\lambda t)^{(y-\lambda) / \lambda} \mid \sum_{\ell \geq 0}^{r} \mathfrak{b}_{\ell} \frac{\lambda^{\ell} t^{\ell}}{\ell !} x^{n}\right\rangle
\end{aligned}
$$

which is equal to

$$
\begin{aligned}
& -\sum_{i=1}^{r} a_{i} D \mathcal{E}_{n-1}(\lambda, y-\lambda \mid \mathbf{a} ; \mathbf{b})+\frac{r}{n} \sum_{\ell=0}^{n}\left(\begin{array}{l}
n \\
\ell
\end{array}\right) \lambda^{\ell} \mathfrak{b}_{\ell} D \mathcal{E}_{n-\ell}(\lambda, y-\lambda \mid \mathbf{a} ; \mathbf{b}) \\
& -\frac{1}{n} \sum_{i=1}^{r} a_{i} \sum_{\ell=0}^{n}\left(\begin{array}{l}
n \\
\ell
\end{array}\right) \lambda^{\ell} \mathfrak{b}_{\ell} D \mathcal{E}_{n-\ell}\left(\lambda, y-\lambda \mid a_{i}, a_{1}, \ldots, a_{r} ; \mathbf{b}\right) .
\end{aligned}
$$

By using (3.4), (3.5) and (3.6) instead of (3.3), (3.2) and (3.1), respectively, we complete the proof. 
Theorem 3.5 For all $n \geq 0$,

$$
\begin{aligned}
& D \mathcal{E}_{n+1}(\lambda, x \mid \mathbf{a} ; \mathbf{b}) \\
& =x D \mathcal{E}_{n}(\lambda, x-\lambda \mid \mathbf{a} ; \mathbf{b})-\sum_{i=1}^{r} a_{i} \sum_{m=0}^{n} S_{1}(n, m) \lambda^{n-m} B E_{m}(x-\lambda \mid \mathbf{a} ; \mathbf{b}) \\
& \quad-\sum_{m=0}^{n} \sum_{\ell=0}^{m} S_{1}(n, m) \lambda^{n-m}\left(\begin{array}{c}
n \\
m
\end{array}\right)\left(\frac{B_{\ell+1}}{\ell+1} \sum_{i=1}^{r} a_{i}^{\ell+1}+\frac{E_{\ell}(1)}{2} \sum_{j=1}^{s} b_{j}^{\ell+1}\right) \\
& \quad \times B E_{m-\ell}(x-\lambda \mid \mathbf{a} ; \mathbf{b}),
\end{aligned}
$$

where $B_{\ell}$ is the $\ell$ th Bernoulli number and $E_{\ell}(1)$ is the $\ell$ th Euler polynomial evaluated at 1.

Proof It is well known that for $s_{n}(x) \sim(g(t), f(t)), s_{n+1}(x)=\left(x-g^{\prime}(t) / g(t)\right) \frac{1}{f^{\prime}(t)} s_{n}(x)$ (see [1, $2])$. In our case, by (1.6), we have

$$
D \mathcal{E}_{n+1}(\lambda, x \mid \mathbf{a} ; \mathbf{b})=x D \mathcal{E}_{n}(\lambda, x-\lambda \mid \mathbf{a} ; \mathbf{b})-e^{-\lambda t} \frac{g^{\prime}(t)}{g(t)} D \mathcal{E}_{n}(\lambda, x \mid \mathbf{a} ; \mathbf{b}),
$$

and by Theorem 2.1, we obtain

$$
\begin{aligned}
D \mathcal{E}_{n+1}(\lambda, x \mid \mathbf{a} ; \mathbf{b})= & x D \mathcal{E}_{n}(\lambda, x-\lambda \mid \mathbf{a} ; \mathbf{b}) \\
& -\sum_{m=0}^{n} S_{1}(n, m) \lambda^{n-m} e^{-\lambda t} \frac{g^{\prime}(t)}{g(t)} B E_{m}(x \mid \mathbf{a} ; \mathbf{b}) .
\end{aligned}
$$

Note that

$$
\begin{aligned}
\frac{g^{\prime}(t)}{g(t)} & =(\log g(t))^{\prime}=\sum_{i=1}^{r} \frac{a_{i} e^{a_{i} t}}{e^{a_{i} t}-1}-\frac{r}{t}+\sum_{j=1}^{s} \frac{b_{j} e^{b_{j} t}}{e^{b_{j} t}+1} \\
& =\sum_{i=1}^{r} a_{i}+\frac{1}{t} \sum_{i=1}^{r}\left(\frac{a_{i} t}{e^{a_{i} t}-1}-1\right)+\frac{1}{2} \sum_{j=1}^{s} \frac{2 b_{j} e^{b_{j} t}}{e^{b_{j} t}+1} \\
& =\sum_{i=1}^{r} a_{i}+\frac{1}{t} \sum_{i=1}^{r} \sum_{\ell \geq 0} \beta_{\ell} a_{i}^{\ell^{\ell}} \frac{t^{\ell}}{\ell !}+\frac{1}{2} \sum_{j=1}^{s} \sum_{\ell \geq 0} E_{\ell}(1) b_{j}^{\ell+1} \frac{t^{\ell}}{\ell !} \\
& =\sum_{i=1}^{r} a_{i}+\sum_{\ell \geq 0} \frac{\beta_{\ell+1}}{(\ell+1) !} \sum_{i=1}^{r} a_{i}^{\ell+1} t^{\ell}+\frac{1}{2} \sum_{\ell \geq 0} \frac{E_{\ell}(1)}{\ell !} \sum_{j=1}^{s} b_{j}^{\ell+1} t^{\ell} .
\end{aligned}
$$

So

$$
\begin{aligned}
\frac{g^{\prime}(t)}{g(t)} B E_{m}(x \mid \mathbf{a} ; \mathbf{b})= & \sum_{i=1}^{r} a_{i} B E_{m}(x \mid \mathbf{a} ; \mathbf{b})+\sum_{\ell=0}^{m}\left(\begin{array}{c}
m \\
\ell
\end{array}\right) \frac{\beta_{\ell+1}}{\ell+1} \sum_{i=1}^{r} a_{i}^{\ell+1} B E_{m-\ell}(x \mid \mathbf{a} ; \mathbf{b}) \\
& +\frac{1}{2} \sum_{\ell=0}^{m}\left(\begin{array}{c}
m \\
\ell
\end{array}\right) E_{\ell}(1) \sum_{j=1}^{s} b_{j}^{\ell+1} B E_{m-\ell}(x \mid \mathbf{a} ; \mathbf{b}) .
\end{aligned}
$$

Hence, by substituting into (3.7), we complete the proof. 


\section{Relations with other families of polynomials}

In this section, we establish a connection between Barnes-type Daehee with $\lambda$-parameter and degenerate Euler mixed-type polynomials and several known families of polynomials.

Theorem 4.1 For all $n \geq 0$,

$$
D \mathcal{E}_{n}(\lambda, x \mid \mathbf{a} ; \mathbf{b})=\sum_{m=0}^{n}\left(\begin{array}{c}
n \\
m
\end{array}\right) D \mathcal{E}_{n-m}(\lambda \mid \mathbf{a} ; \mathbf{b})(x \mid \lambda)_{m}
$$

Proof Note that $(x \mid \lambda)_{n} \sim\left(1, \frac{e^{\lambda t}-1}{\lambda}\right)$. Let $D \mathcal{E}_{n}(\lambda, x \mid \mathbf{a} ; \mathbf{b})=\sum_{m=0}^{n} c_{n, m}(x \mid \lambda)_{m}$. By (1.3) and (1.6), we have

$$
\begin{aligned}
c_{n, m} & =\frac{1}{m !}\left\langle P_{r, s}(t) \mid t^{m} x^{n}\right\rangle=\left(\begin{array}{c}
n \\
m
\end{array}\right)\left\langle P_{r, s}(t) \mid x^{n-m}\right\rangle \\
& =\left(\begin{array}{c}
n \\
m
\end{array}\right) D \mathcal{E}_{n-m}(\lambda \mid \mathbf{a} ; \mathbf{b}),
\end{aligned}
$$

which completes the proof.

For the following, we note that $B_{n}^{(\alpha)}(x) \sim\left(\frac{\left(e^{t}-1\right)^{\alpha}}{t^{\alpha}}, t\right)$.

Theorem 4.2 For all $n \geq 0$, the polynomial $D \mathcal{E}_{n}(\lambda, x \mid \mathbf{a} ; \mathbf{b})$ is given by

$$
\sum_{m=0}^{n}\left(\sum_{\ell=m}^{n} \sum_{k=0}^{n-\ell} \sum_{q=0}^{n-\ell-k} \sum_{p=0}^{q} \frac{\left(\begin{array}{c}
n \\
\ell
\end{array}\right)\left(\begin{array}{c}
n-\ell \\
k
\end{array}\right)\left(\begin{array}{c}
n-\ell-k \\
q
\end{array}\right)}{\left(\begin{array}{c}
q+\alpha \\
\alpha
\end{array}\right)} a_{\ell, k, q, p} D \mathcal{E}_{n-\ell-k-q}(\lambda \mid \mathbf{a} ; \mathbf{b})\right) B_{m}^{(\alpha)}(x),
$$

where $a_{\ell, k, q, p}=S_{1}(\ell, m) S_{1}(q+\alpha, q-p+\alpha) S_{2}(q-p+\alpha, \alpha) \lambda^{k+\ell+p-m} b_{\ell}^{(\alpha)}$ and $b_{\ell}^{(\alpha)}$ is the th Bernoulli number of the second kind of order $\alpha$ given by $\left(\frac{t}{\log (1+t)}\right)^{\alpha}=\sum_{\ell \geq 0} b_{\ell}^{(\alpha)} \frac{t^{\ell}}{k !}$.

Proof Let $D \mathcal{E}_{n}(\lambda, x \mid \mathbf{a} ; \mathbf{b})=\sum_{m=0}^{n} c_{n, m} B_{m}^{(\alpha)}(x)$. By (1.3) and (1.6), we have

$$
\begin{aligned}
c_{n, m} & =\frac{1}{m ! \lambda^{m}}\left\langle P_{r, s}(t)\left(\frac{(1+\lambda t)^{1 / \lambda}-1}{t}\right)^{\alpha}\left(\frac{\lambda t}{\log (1+\lambda t)}\right)^{\alpha} \mid(\log (1+\lambda t))^{m} x^{n}\right\rangle \\
& =\frac{1}{\lambda^{m}} \sum_{\ell=m}^{n}\left(\begin{array}{l}
n \\
\ell
\end{array}\right) \lambda^{\ell} S_{1}(\ell, m)\left\langle P_{r, s}(t)\left(\frac{(1+\lambda t)^{1 / \lambda}-1}{t}\right)^{\alpha} \mid\left(\frac{\lambda t}{\log (1+\lambda t)}\right)^{\alpha} x^{n-\ell}\right\rangle \\
& =\frac{1}{\lambda^{m}} \sum_{\ell=m}^{n} \sum_{k=0}^{n-\ell}\left(\begin{array}{c}
n \\
\ell
\end{array}\right)\left(\begin{array}{c}
n-\ell \\
k
\end{array}\right) S_{1}(\ell, m) \lambda^{\ell+k} b_{k}^{(\alpha)}\left\langle P_{r, s}(t)\left(\frac{(1+\lambda t)^{1 / \lambda}-1}{t}\right)^{\alpha} \mid x^{n-\ell-k}\right\rangle .
\end{aligned}
$$

One can show that

$$
\begin{aligned}
\left(\frac{(1+\lambda t)^{1 / \lambda}-1}{t}\right)^{\alpha} & =\left(\frac{e^{\frac{1}{\lambda} \log (1+\lambda t)}-1}{t}\right)^{\alpha} \\
& =\sum_{q \geq 0} \sum_{p=0}^{q}\left(\begin{array}{c}
q+\alpha \\
\alpha
\end{array}\right)^{-1} S_{1}(q+\alpha, q-p+\alpha) S_{2}(q-p+\alpha, \alpha) \lambda^{p} \frac{t^{q}}{q !}
\end{aligned}
$$


where $S_{2}(n, m)$ is the Stirling number of the second kind. Thus,

$$
\begin{aligned}
& \left\langle P_{r, s}(t)\left(\frac{(1+\lambda t)^{1 / \lambda}-1}{t}\right)^{\alpha} \mid x^{n-\ell-k}\right\rangle \\
& =\sum_{q=0}^{n-\ell-k} \sum_{p=0}^{q} \frac{\left(\begin{array}{c}
n-\ell-k \\
q
\end{array}\right)}{\left(\begin{array}{c}
q+\alpha \\
\alpha
\end{array}\right)} S_{1}(q+\alpha, q-p+\alpha) S_{2}(q-p+\alpha, \alpha) \lambda^{p}\left\langle P_{r, s}(t) \mid x^{n-\ell-k-q}\right\rangle,
\end{aligned}
$$

where $\left\langle P_{r, s}(t) \mid x^{n-\ell-k-q}\right\rangle=D \mathcal{E}_{n-\ell-k-q}(\lambda \mid \mathbf{a} ; \mathbf{b})$. Hence,

$$
c_{n, m}=\sum_{\ell=m}^{n} \sum_{k=0}^{n-\ell} \sum_{q=0}^{n-\ell-k} \sum_{p=0}^{q} \frac{\left(\begin{array}{c}
n \\
\ell
\end{array}\right)\left(\begin{array}{c}
n-\ell \\
k
\end{array}\right)\left(\begin{array}{c}
n-\ell-k \\
q
\end{array}\right)}{\left(\begin{array}{c}
q+\alpha \\
\alpha
\end{array}\right)} a_{\ell, k, q, p} D \mathcal{E}_{n-\ell-k-q}(\lambda \mid \mathbf{a} ; \mathbf{b}),
$$

which completes the proof.

By similar techniques as in the proof of the last theorem, we can express our polynomials $D \mathcal{E}_{n}(\lambda, x \mid \mathbf{a} ; \mathbf{b})$ in terms of the degenerate Bernoulli polynomials $\beta_{n}^{(\alpha)}(\lambda, x)$ of order $\alpha$. These polynomials are the Sheffer sequence which is given by $\beta_{n}^{(\alpha)}(\lambda, x) \sim\left(\left(\frac{\lambda\left(e^{t}-1\right)}{e^{\lambda t}-1}\right)^{\alpha}, \frac{e^{\lambda t}-1}{\lambda}\right)$.

Theorem 4.3 For all $n \geq 0$, the polynomial $D \mathcal{E}_{n}(\lambda, x \mid \mathbf{a} ; \mathbf{b})$ is given by

$$
\sum_{m=0}^{n}\left(\begin{array}{c}
n \\
m
\end{array}\right) c_{n, m} \beta_{m}^{(\alpha)}(\lambda, x)
$$

where $c_{n, m}=\sum_{q=0}^{n-m} \sum_{p=0}^{q} \frac{\left(\begin{array}{c}n-m \\ q\end{array}\right)}{\left(\begin{array}{c}q+\alpha \\ \alpha\end{array}\right)} S_{1}(q+\alpha, q-p+\alpha) S_{2}(q-p+\alpha, \alpha) \lambda^{p} D \mathcal{E}_{n-m-q}(\lambda \mid \mathbf{a} ; \mathbf{b})$.

Now we are interested in expressing our polynomials in terms of $H_{n}^{(\alpha)}(x \mid \mu)$ which are called the Frobenius-Euler polynomials of order $\alpha$. Note that $H_{n}^{(\alpha)}(x \mid \mu) \sim\left(\left(\frac{e^{t}-\mu}{1-\mu}\right)^{\alpha}, t\right)$ (see $[10,24])$.

Theorem 4.4 For all $n \geq 0$,

$$
D \mathcal{E}_{n}(\lambda, x \mid \mathbf{a} ; \mathbf{b})=\sum_{m=0}^{n}\left(\frac{a_{n, m}}{(1-\mu)^{\alpha} \lambda^{m}}\right) H_{m}^{(\alpha)}(x \mid \mu)
$$

where

$$
a_{n, m}=\sum_{\ell=m}^{n} \sum_{k=0}^{n-\ell} \sum_{p=0}^{\alpha}\left(\begin{array}{l}
n \\
\ell
\end{array}\right)\left(\begin{array}{c}
n-\ell \\
k
\end{array}\right)\left(\begin{array}{l}
\alpha \\
p
\end{array}\right) S_{1}(\ell, m) \lambda^{\ell}(-\mu)^{\alpha-p} D \mathcal{E}_{k}(\lambda \mid \mathbf{a} ; \mathbf{b})(p \mid \lambda)_{n-\ell-k} .
$$

Proof Let $D \mathcal{E}_{n}(\lambda, x \mid \mathbf{a} ; \mathbf{b})=\sum_{m=0}^{n} c_{n, m} H_{m}^{(\alpha)}(x \mid \mu)$. By (1.3) and (1.6), we have

$$
\begin{aligned}
c_{n, m} & =\frac{1}{m !(1-\mu)^{\alpha} \lambda^{m}}\left\langle P_{r, s}(t)\left((1+\lambda t)^{1 / \lambda}-\mu\right)^{\alpha} \mid(\log (1+\lambda t))^{m} x^{n}\right\rangle \\
& =\frac{1}{m !(1-\mu)^{\alpha} \lambda^{m}}\left\langle P_{r, s}(t)\left((1+\lambda t)^{1 / \lambda}-\mu\right)^{\alpha} \mid m ! \sum_{\ell \geq m} S_{1}(\ell, m) \frac{\lambda^{\ell}}{\ell !} t^{\ell} x^{n}\right\rangle
\end{aligned}
$$




$$
\begin{aligned}
& \left.=\frac{1}{(1-\mu)^{\alpha} \lambda^{m}} \sum_{\ell=m}^{n}\left(\begin{array}{l}
n \\
\ell
\end{array}\right) S_{1}(\ell, m) \lambda^{\ell}\left|\left((1+\lambda t)^{1 / \lambda}-\mu\right)^{\alpha}\right| P_{r, s}(t) x^{n-\ell}\right\rangle \\
& =\frac{1}{(1-\mu)^{\alpha} \lambda^{m}} \sum_{\ell=m}^{n} \sum_{k=0}^{n-\ell}\left(\begin{array}{c}
n \\
\ell
\end{array}\right)\left(\begin{array}{c}
n-\ell \\
k
\end{array}\right) S_{1}(\ell, m) \lambda^{\ell} D \mathcal{E}_{k}(\lambda \mid \mathbf{a} ; \mathbf{b}) w_{n, \ell, k},
\end{aligned}
$$

where

$$
\begin{aligned}
w_{n, \ell, k} & =\left\langle\left((1+\lambda t)^{1 / \lambda}-\mu\right)^{\alpha} \mid x^{n-\ell-k}\right\rangle \\
& =\left\langle\sum_{p=0}^{\alpha}\left(\begin{array}{l}
\alpha \\
p
\end{array}\right)(-\mu)^{\alpha-p}(1+\lambda t)^{p / \lambda} \mid x^{n-\ell-k}\right\rangle \\
& =\sum_{p=0}^{\alpha}\left(\begin{array}{l}
\alpha \\
p
\end{array}\right)(-\mu)^{\alpha-p}\left\langle\sum_{q \geq 0}(p \mid \lambda)_{q} \frac{t^{q}}{q !} \mid x^{n-\ell-k}\right\rangle \\
& =\sum_{p=0}^{\alpha}\left(\begin{array}{l}
\alpha \\
p
\end{array}\right)(-\mu)^{\alpha-p}(p \mid \lambda)_{n-\ell-k} .
\end{aligned}
$$

Thus, the constants $c_{n, m}$ are given by

$$
\frac{1}{(1-\mu)^{\alpha} \lambda^{m}} \sum_{\ell=m}^{n} \sum_{k=0}^{n-\ell} \sum_{p=0}^{\alpha}\left(\begin{array}{c}
n \\
\ell
\end{array}\right)\left(\begin{array}{c}
n-\ell \\
k
\end{array}\right)\left(\begin{array}{c}
\alpha \\
p
\end{array}\right) S_{1}(\ell, m) \lambda^{\ell}(-\mu)^{\alpha-p} D \mathcal{E}_{k}(\lambda \mid \mathbf{a} ; \mathbf{b})(p \mid \lambda)_{n-\ell-k},
$$

which completes the proof.

Now we are interested in expressing our polynomials in terms of $\mathcal{E}_{n}^{(\alpha)}(\lambda, x)$ which are called the degenerate Euler polynomials of order $\alpha$. Note that

$$
\mathcal{E}_{n}^{(\alpha)}(\lambda, x) \sim\left(\left(\frac{e^{t}+1}{2}\right)^{\alpha}, \frac{e^{\lambda t}-1}{\lambda}\right)
$$

(see [10]). Using similar techniques as in the proof of the above theorem, we obtain the following relation.

Theorem 4.5 For all $n \geq 0$, the polynomial $D \mathcal{E}_{n}(\lambda, x \mid \mathbf{a} ; \mathbf{b})$ is given by

$$
\frac{1}{2^{\alpha}} \sum_{m=0}^{n}\left(\begin{array}{c}
n \\
m
\end{array}\right)\left(\sum_{q=0}^{n-m} \sum_{p=0}^{\alpha}\left(\begin{array}{c}
n-m \\
q
\end{array}\right)\left(\begin{array}{l}
\alpha \\
p
\end{array}\right)(p \mid \lambda)_{q} D \mathcal{E}_{n-m-q}(\lambda \mid \mathbf{a} ; \mathbf{b})\right) \mathcal{E}_{m}^{(\alpha)}(\lambda, x) .
$$

\section{Competing interests}

The authors declare that they have no competing interests.

\section{Authors' contributions}

All authors contributed equally and significantly in writing this article. All authors read and approved the final manuscript.

\section{Author details}

${ }^{1}$ Institute of Natural Sciences, Far Eastern Federal University, Vladivostok, 690950, Russia. ²Department of Mathematics, Sogang University, Seoul, 121-742, South Korea. ${ }^{3}$ Department of Mathematics, Kwangwoon University, Seoul, South

Korea. ${ }^{4}$ Department of Mathematics, University of Haifa, Haifa, 3498838, Israel.

Received: 20 February 2015 Accepted: 24 April 2015 Published online: 08 May 2015 
References

1. Roman, S: More on the umbral calculus, with emphasis on the $q$-umbral calculus. J. Math. Anal. Appl. 107, 222-254 (1985)

2. Roman, S: The Umbral Calculus. Dover, New York (2005)

3. Kim, DS, Kim, T, Kwon, HI, Mansour, T: Barnes-type degenerate Bernoulli and Euler mixed-type polynomials (submitted)

4. Kim, DS, Kim, T, Kwon, HI, Mansour, T, Seo, JJ: Barnes-type Peters polynomial with umbral calculus viewpoint. J. Inequal. Appl. 2014, 324 (2014)

5. Kim, DS, Kim, T, Kwon, HI, Mansour, T: Barnes-type Narumi of the first kind and poly-Cauchy of the first kind mixed-type polynomials. Adv. Stud. Theor. Phys. 8(22), 961-975 (2014)

6. Kim, DS, Kim, T, Lee, S-H, Seo, JJ: A note on the lambda-Daehee polynomials. Int. J. Math. Anal. 7(62), 3069-3080 (2013)

7. Park, J-W: On the twisted Daehee polynomials with q-parameter. Adv. Differ. Equ. 2014, 304 (2014)

8. Carlitz, L, Stirling, D: Bernoulli and Eulerian numbers. Util. Math. 15, 51-88 (1979)

9. Carlitz, L: A degenerate Staudt-Clausen theorem. Arch. Math. (Basel) 7, 28-33 (1956)

10. Kim, DS, Kim, T: Higher-order degenerate Euler polynomials. Appl. Math. Sci. (Ruse) 9(2), 57-73 (2015)

11. Kim, DS, Kim, T: Some identities of degenerate Euler polynomials arising from fermionic integral on $Z_{p}$. Integral Transforms Spec. Funct. 26(4), 295-302 (2015)

12. Araci, S, Acikgoz, M, Sen, E: On the extended Kim's p-adic $q$-deformed fermionic integrals in the $p$-adic integer ring. J. Number Theory 133(10), 3348-3361 (2013)

13. Hwang, K-W, Dolgy, DV, Kim, DS, Kim, T, Lee, SH: Some theorems on Bernoulli and Euler numbers. Ars Comb. 109 285-297 (2013)

14. Kim, DS, Kim, T: q-Bernoulli polynomials and q-umbral calculus. Sci. China Math. 57(9), 1867-1874 (2014)

15. Kim, T: Some identities on the $q$-Euler polynomials of higher order and $q$-Stirling numbers by the fermionic $p$-adic integral on $Z_{p}$. Russ. J. Math. Phys. 16(4), 484-491 (2009)

16. Luo, Q-M, Qi, F: Relationships between generalized Bernoulli numbers and polynomials and generalized Euler numbers and polynomials. Adv. Stud. Contemp. Math. (Kyungshang) 7(1), 11-18 (2003)

17. Ozden, $\mathrm{H}$ : p-Adic distribution of the unification of the Bernoulli, Euler and Genocchi polynomials. Appl. Math. Comput. 218(3), 970-973 (2011)

18. Park, J-W, Rim, S-H, Kwon, J: The twisted Daehee numbers and polynomials. Adv. Differ. Equ. 2014, 1 (2014)

19. Rim, S-H, Joung, J, Jin, J-H, Lee, S-J: A note on the weighted Carlitz's type $q$-Euler numbers and $q$-Bernstein polynomials. Proc. Jangjeon Math. Soc. 15(2), 195-201 (2012)

20. Zhang, Z, Yang, H: Some closed formulas for generalized Bernoulli-Euler numbers and polynomials. Proc. Jangjeon Math. Soc. 11(2), 191-198 (2008)

21. Kim, DS, Kim, T, Kwon, HI, Seo, JJ: Identities of some special mixed-type polynomials. Adv. Stud. Theor. Phys. 8(17), 745-754 (2014)

22. Lim, D, Do, Y: Some identities of Barnes-type special polynomials. Adv. Differ. Equ. 2015, 42 (2015)

23. Kim, DS, Kim, T, Dolgy, DV, Komtasu, T: Barnes-type degenerate Bernoulli polynomials. Adv. Stud. Contemp. Math. 25, $121-146(2015)$

24. Kurt, B, Simsek, Y: On the generalized Apostol-type Frobenius-Euler polynomials. Adv. Differ. Equ. 2013,1 (2013)

\section{Submit your manuscript to a SpringerOpen ${ }^{\circ}$ journal and benefit from:}

- Convenient online submission

Rigorous peer review

- Immediate publication on acceptance

- Open access: articles freely available online

- High visibility within the field

- Retaining the copyright to your article 